\title{
An evaluation of diffraction peak profile analysis (DPPA) methods to study plastically deformed metals
}

DOI:

10.1016/j.matdes.2016.08.091

\section{Document Version}

Accepted author manuscript

Link to publication record in Manchester Research Explorer

\section{Citation for published version (APA):}

Simm, T. H., Withers, P., \& Quinta Da Fonseca, J. (2016). An evaluation of diffraction peak profile analysis (DPPA) methods to study plastically deformed metals. Materials \& Design, 111.

https://doi.org/10.1016/j.matdes.2016.08.091

\section{Published in:}

Materials \& Design

\section{Citing this paper}

Please note that where the full-text provided on Manchester Research Explorer is the Author Accepted Manuscript or Proof version this may differ from the final Published version. If citing, it is advised that you check and use the publisher's definitive version.

\section{General rights}

Copyright and moral rights for the publications made accessible in the Research Explorer are retained by the authors and/or other copyright owners and it is a condition of accessing publications that users recognise and abide by the legal requirements associated with these rights.

\section{Takedown policy}

If you believe that this document breaches copyright please refer to the University of Manchester's Takedown Procedures [http://man.ac.uk/04Y6Bo] or contact uml.scholarlycommunications@manchester.ac.uk providing relevant details, so we can investigate your claim.

\section{OPEN ACCESS}




\section{Accepted Manuscript}

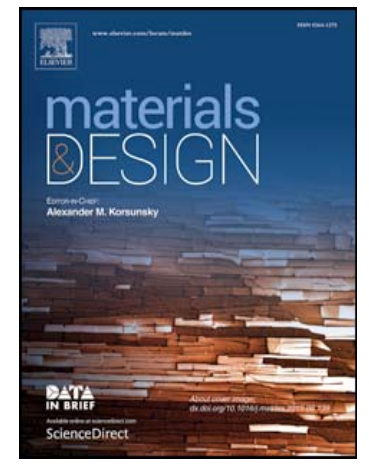

An evaluation of diffraction peak profile analysis (DPPA) methods to study plastically deformed metals

T.H. Simm, P.J. Withers, J. Quinta da Fonseca

PII:

S0264-1275(16)31154-6

DOI: doi: $10.1016 /$ j.matdes.2016.08.091

Reference: $\quad$ JMADE 2244

To appear in:

Received date: 13 July 2016

Revised date: $\quad 30$ August 2016

Accepted date: $\quad 31$ August 2016

Please cite this article as: T.H. Simm, P.J. Withers, J. Quinta da Fonseca, An evaluation of diffraction peak profile analysis (DPPA) methods to study plastically deformed metals, (2016), doi: 10.1016/j.matdes.2016.08.091

This is a PDF file of an unedited manuscript that has been accepted for publication. As a service to our customers we are providing this early version of the manuscript. The manuscript will undergo copyediting, typesetting, and review of the resulting proof before it is published in its final form. Please note that during the production process errors may be discovered which could affect the content, and all legal disclaimers that apply to the journal pertain. 


\title{
An evaluation of Diffraction Peak Profile Analysis (DPPA) methods to study plastically deformed metals
}

\author{
T.H. Simm ${ }^{a, b *}$, P.J. Withers ${ }^{a}$, J. Quinta da Fonseca ${ }^{a}$ \\ a) Manchester Materials Science Centre, The University of Manchester, Grosvenor Street, Manchester M1 7HS, UK. b) Institute of Structural Materials, \\ Swansea University, Bay Campus, SA1 8EN, UK. *Correspondence author: Thomas.Simm@dMata.co.uk
}

Key words: Warren-Averbach; diffraction peak profile analysis; $x$-ray diffraction; titanium; stainless steel; Williamson-Hall

\section{Abstract}

A range of diffraction peak profile analysis (DPPA) techniques were used to determine details of the microstructure of plastically deformed alloys. Four different alloys were deformed by uniaxial tension and compression to a range of strains. The methods we have considered include, the full-width, Williamson-Hall methods, Warren-Averbach methods, and van Berkum's alternative method. Different metals were chosen to understand the effect of the deformation microstructure and crystal structure, a nickel alloy, two stainless steel alloys and two titanium alloys.

The dislocation density values found by Williamson-Hall and Warren-Averbach methods were found to be close to those expected from TEM results of similar metals. When using the Warren-Averbach methods the results suggest that systematic errors in the dislocation density are introduced by three main factors: (1) separation of instrumental broadening, (2) separation of size and strain broadening, and (3) separation of dislocation density and arrangement. Which suggests in many cases the simpler Williamson-Hall method may be preferable.

The other main parameters that can be determined by DPPA are the crystal size and the dislocation arrangement. The work suggests that further investigation is needed to understand what use if any these parameters have for quantifying the deformed microstructure of plastically deformed metals.

\section{Introduction}

The study of the shape of diffraction peaks is a well-developed and valuable method for the study of the microstructure of crystalline materials [1-4]. This technique, which we refer to as Diffraction Peak Profile Analysis (DPPA), is used to find details of the microstructure such as the crystal or subgrain size, the dislocation density and other defects, and the slip systems present. DPPA is an indirect and statistical characterisation method that uses information from a diffraction pattern which consists of information from many grains. Because of this it is necessary to make approximations as to how the peaks should broaden by the microstructures. There are a number of different DPPA methods that can be used $[3,5,6]$, each method has its own set of assumptions and should therefore yield different results. But because research is often focused on model development or on applying one technique to one sample, there has been a lack of 
research on comparing methods and establishing how useful they are. The main problem with evaluating the methods is due to the difficulty in verifying the results by other means. The only parameters that are adequately measured by both DPPA and other methods are the dislocation density and the crystal size. For the dislocation density the results show some agreement [7-10]. Whereas for the crystal size, the values show agreement for un-deformed samples [11-13], but for sample with strain present such as deformed samples there can be problems, and in most cases the sizes are significantly smaller than found by other methods $[1,11,14,15]$.

In order to address these issues a number of alloys have been studied, nickel-200, stainless steel 316, stainless steel $304, \mathrm{Ti}-6 \mathrm{Al}-4 \mathrm{~V}$ and Ti-CP. The alloys used were chosen because they had a relatively simple microstructure but deform in different ways. The stacking fault energy (SFE) of a metal has a large influence on the deformed microstructure. We investigate this by using the nickel alloy, which has a high SFE and develops a cell structure, and the stainless steel alloys, which have a low SFE and do not develop a defined cell structure. In other regards the alloys are similar, they all have a face-centred-cubic (FCC) crystal structure, a similar grain size, they all work-harden and have similar elastic constants [16]. The two different types of stainless steel were used because they are expected to have similar deformation microstructures. Two titanium alloys, Ti-6Al-4V and Ti-CP, are investigated to determine the influence of a HCP crystal structure on the results of the DPPA methods. An additional reason for the choice of these alloys is that their deformation microstructure has been well researched [17-22].

We will consider a number of commonly used DPPA methods. These include using the full-width on its own [23-25], the Williamson-Hall method [5,26,27], the Warren-Averbach method [28-31] and the alternative method [14]. We will use these methods on the different alloys deformed by uni-axial compression and tension to different applied strains. The use of different metals deformed to different amounts of plastic deformation allows a broad evaluation of the techniques.

\section{Materials and Methods}

The compositions of the alloys studied are shown in Table 1. All alloys were tested after annealing so that they have minimal residual stresses (between different grains and within grains), dislocations, other defects (e.g. planar faults) and precipitates. The alloys either consist of a single phase, or in the case of Ti-6Al-4V a dominant phase; and they all have a relatively large grain size, $\sim 30 \mu \mathrm{m}$, with a near random texture. The alloys were deformed at room-temperature by uni-axial tension and compression to a range of strains up to 0.3. The titanium alloys and SS-304 were tested by tension; for SS-304 the samples had a gauge diameter of $6 \mathrm{~mm}$ and parallel length of $35 \mathrm{~mm}$, and for titanium alloys the gauge diameter was $8 \mathrm{~mm}$ and the parallel length $48 \mathrm{~mm}$. The other alloys were tested by compression from cylinders with diameter of approximately $20 \mathrm{~mm}$ and length $20 \mathrm{~mm}$. Mechanical tests were conducted with a constant cross-head speed, the initial strain rate was $0.1 \mathrm{~mm} / \mathrm{min}$ for SS-304, $2 \mathrm{~mm} / \mathrm{min}$ for SS-316 and Ni-200, and $1 \mathrm{~mm} / \mathrm{min}$ for the titanium alloys. For hardness tests a Vickers micro-hardness testing machine was used with a force of $4.9 \mathrm{~N}(0.5 \mathrm{~kg})$, 
each result is the average of approximately 10 different measurements. The samples were prepared in the same way as for laboratory $\mathrm{X}$-ray diffraction (see below).

The titanium alloys were measured by synchrotron $\mathrm{x}$-rays and the other alloys by laboratory X-rays. For laboratory X-ray measurements, a Philips X-pert X-ray diffractometer at the Materials Science Centre, Manchester, was used. Co-K $\alpha$ radiation was used instead of copper because it does not cause fluorescence in steel samples. The samples were scanned between angles of $40^{\circ}$ and $130^{\circ}$ (in $2 \theta$ ) to obtain five FCC peaks: $111,200,220,311$ and 222. Two step sizes were used $0.5^{\circ}\left(g^{\sim} 0.005 \AA^{-1}\right)$ around the FCC diffraction peaks and $0.015^{\circ}\left(g^{\sim} 1.7 \times 10^{-4} \AA^{-1}\right)$ at other points to determine the background. An annealed SS-316 sample was used as the instrumental standard; this was chosen over a silicon standard because it had the same amount of broadening as a silicon standard and the peaks were in the same position as those measured. This latter factor is important because the instrumental broadening changes with $2 \theta$ and so the sample used was thought to provide a more realistic measure of the instrumental broadening. The set-up is a compromise between quality of data and time, one sample took approximately 24 hours, but would be quicker in newer diffractometers. An example diffraction pattern is shown in Figure 1. Flat samples of approximately $1 \mathrm{~cm}^{2}$ were prepared by cutting, grinding and polishing, with a final polish using colloidal silica (particle size 0.05 $\mu \mathrm{m})$. Because of the size of samples, the plane normal of SS-316 and Ni samples was parallel to the tensile direction, and perpendicular to the tensile direction for SS-304.
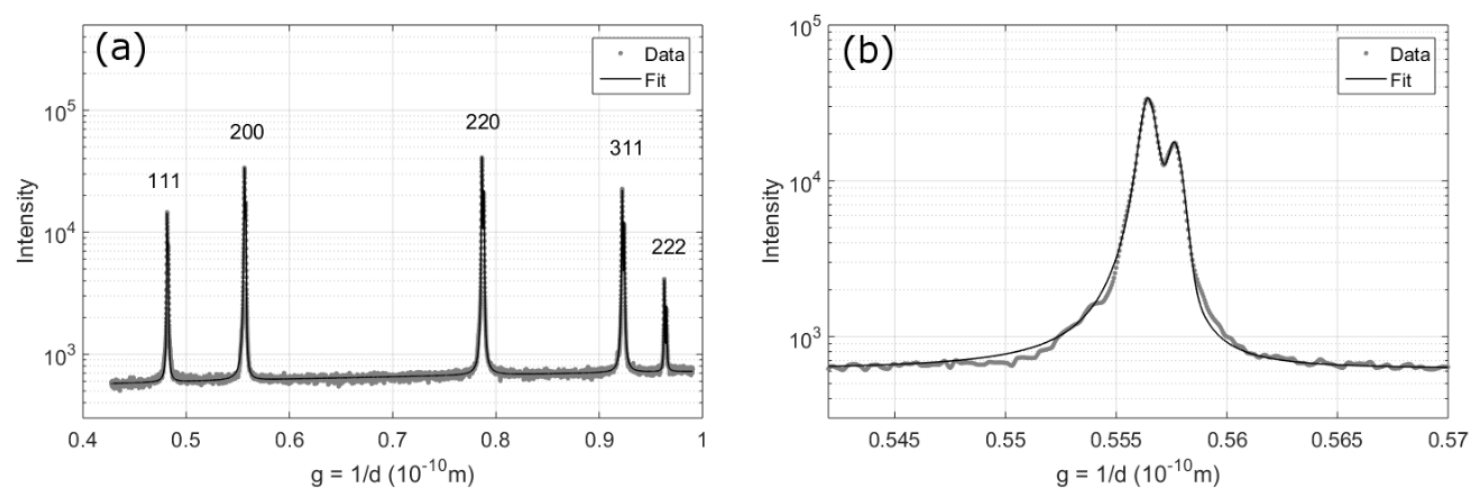

Figure 1, diffraction patterns produced by laboratory $x$-ray. The diffraction pattern is for the SS304 instrumental profile (a) and the pseudo-Voigt fit of one of the peaks (b).
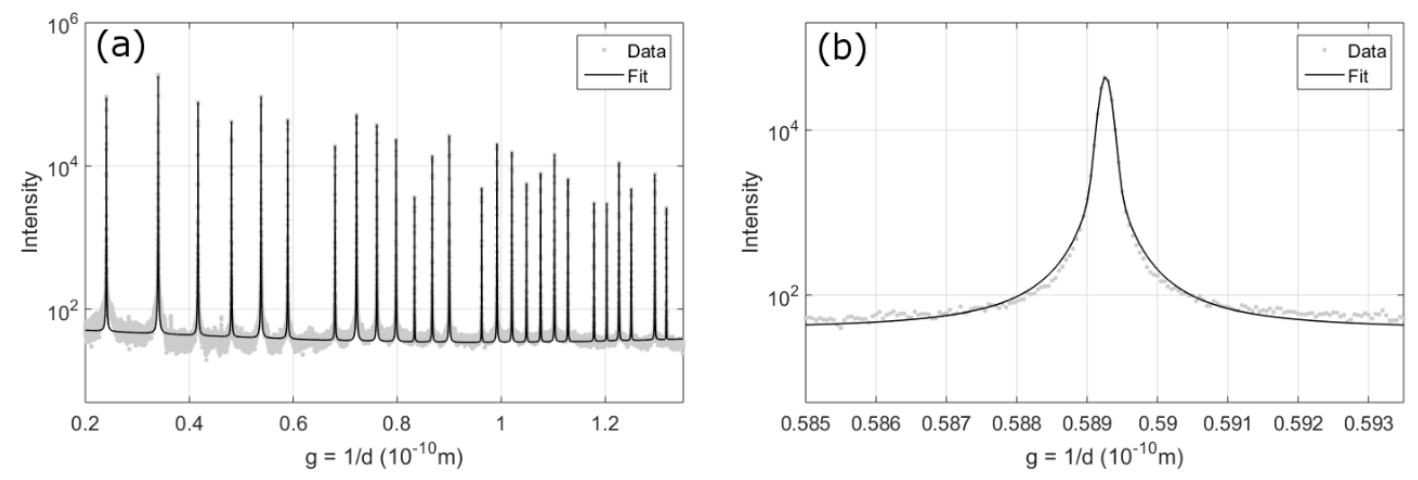
Figure 2, diffraction patterns produced at ID31, ESRF of a LaB6 standard instrumental profile (a) and the pseudo-Voigt fit of one of the peaks (b).

Synchroton diffraction measurements were obtained from the ID31 beamline at ESRF, Grenoble, France. A beam energy of $31.1 \mathrm{keV}$ was used with a wavelength of $0.39827 \AA$, the beam size was set slightly larger than the sample and the position of the sample was moved to obtain the maximum diffracted intensity. To improve the quality of patterns an analyser crystal was used along with all 8 available detectors. Diffraction patterns were obtained by changing $2 \theta$ between $0^{\circ}(g=0)$ and $38^{\circ}(g=1.63 \AA)$, with steps of $0.003^{\circ}$ in $2 \theta$ $\left(g=1.3 \times 10^{-5} \AA\right)$, to allow the assessment of the first 27 hexagonal peaks for titanium. A LaB 6 instrumental sample with dimensions similar to the titanium samples was also measured but with a lower step size of $0.001^{\circ}$ in $2 \theta$. Two separate measurements were taken for each sample, with the diffraction vector either parallel or perpendicular to the tensile direction, and the results presented are the averaged of results from both measurements. An example diffraction pattern is shown in Figure 2. The samples used were square rods of $0.8 \mathrm{~mm}$ wide and $30 \mathrm{~mm}$ long and placed in glass capillaries. They were cut from the centre of the gauge volume parallel to the tensile direction. To minimise any deformation that may be introduced by making the rods they were ground and then wet etched with a solution of hydrochloric acid, hydrofluoric acid, nitric acid and water solution (ratios 30:10:10:50).

Table 1, Composition of the different alloys studied.

\begin{tabular}{|l|l|}
\hline Alloy & Composition in wt.\% \\
\hline SS-304 & $18.2 \mathrm{Cr}, 8.6 \mathrm{Ni}, 0.45 \mathrm{Si}, 1.4 \mathrm{Mn}, 0.05 \mathrm{C}, 0.005 \mathrm{~S}, 0.038 \mathrm{~N}, \mathrm{Fe}$ Bal. \\
\hline SS-316 & $18.1 \mathrm{Cr}, 12.2 \mathrm{Ni}, 3.0 \mathrm{Mo}, \mathrm{Fe}$ Bal. \\
\hline Ni-200 & $0.2 \mathrm{Cu}, 0.2 \mathrm{Fe}, 0.2 \mathrm{Mn}, 0.1 \mathrm{C}, 0.2 \mathrm{Si}, \mathrm{Ni}$ Bal. \\
\hline Ti-6Al-4V & $6.0 \mathrm{Al}, 4.0 \mathrm{~V}, 0.2 \mathrm{Fe}, 0.1 \mathrm{O}, \mathrm{Ti} \mathrm{Bal}$. \\
\hline Ti-CP Grade 2 & $\mathrm{~N} 0.03, \mathrm{C} 0.1, \mathrm{H} 0.015, \mathrm{Fe} 0.3, \mathrm{O} 0.25, \mathrm{Ti}$ Bal. \\
\hline
\end{tabular}

Stress-strain curves for the alloys studied are shown in Figure 3a. All the metals work-harden with a falling work-hardening rate with applied strain, but have different yield and ultimate strengths (although the ultimate strength was not obtained here). The stress-strain curves are important in this work because they can be compared directly with the results of DPPA methods by the use of a relationship between the flow stress ( $\sigma$, the stress needed to allow plastic deformation) and the dislocation density $(\rho)$ [32-34]. A similar relationship has been found for the change in dislocation cell size $(D)[35,36]$. In Figure $3 b$ the change in the hardness of the different alloys with applied strain is shown. The curves display a number of similar features to the stress-strain curves, including their relative magnitude and changes. The main exceptions to this is the constant hardness of Ti-6Al-4V and the relative magnitudes of SS-304 and SS-316. 

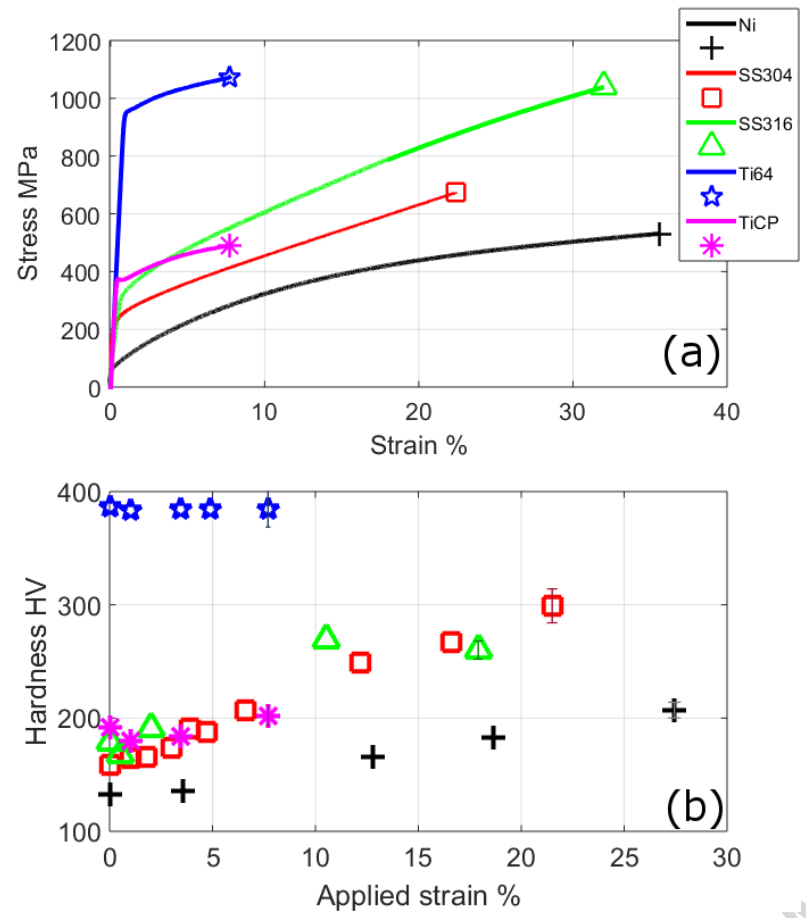

Figure 3, true stress-true strain curves for the different metals studied (a). And the change in Vickers Hardness values (HV) for the different metals studied using $0.5 \mathrm{~kg}$ indent, with engineering strain. Error bars from the average standard deviation of measurements of an alloy are shown with the final marker.

\section{DPPA Methods}

All analysis was conducted using a Matlab GUI built for peak profile analysis (www.dMata.co.uk/dippa). The GUI is available open source and at http://github.com/dMaterialia/DPPA[37], where issues and recommendations for improvement can be reported along with some of the data used in this report. The GUI has two parts, 1) BIGdippa for fitting the peaks and 2) dippaFC for Fourier methods. More details on the analytical procedure can be found in the appendix and elsewhere [38].

In BIGdippa the data is converted to $g$-scale $(g=1 / d=2 \sin \vartheta / \lambda)$ with a constant step size using interpolation. This makes the data easier to work with, particularly when using Fourier methods. The measured diffraction patterns are fitted with a pseudo-Voigt [39] curve for each peak and a quadratic polynomial for the background. A split pseudo-Voigt is used to account for asymmetry of peaks by allowing the full-width and mixing parameter to vary on the left and right sides of the peak. The value of the mixing parameter $(\eta)$ was allowed to change between 0 and 1.2, this allows the possibility of super-Cauchy, or super-Lorentzian, peaks when $\eta>1$. Laboratory $\mathrm{X}$-ray measurements from $\mathrm{K}-\alpha$ radiation consists of two main components, known as $\alpha_{1}$ and $\alpha_{2}$. We account for this in the fitting by assuming the $\alpha_{1}$ and $\alpha_{2}$ have the same shape and fit a diffraction peak to the sum of the two pseudo-Voigt curves. Although, no analytical function can fully describe the peak shape this fitting method provides a good approximation to the peak shapes (Figure 1, Figure 2). To account for instrumental broadening different approaches were taken depending on the DPPA method used. For the full-width and Williamson-Hall methods we assume the peaks are Cauchy; the measured peak $(M)$ is then the sum of the instrumental $(I)$ and physically broadened $(P)$ peaks, 
$\beta_{M}(g)=\beta_{I}+\beta_{P}$, where the full-width is used for $\beta$. This introduces some uncertainty as the peaks are never exactly Cauchy $[27,40]$. To remove the instrumental broadening for Fourier methods the Stokes correction [41] was used.

The fitting in BIGdippa is used directly for analytical methods that use the pseudo-Voigt (e.g. FW and Williamson-Hall method). But is also used indirectly by the Fourier methods to remove overlapping peaks, find the position of the peak's centre and determine background level. In addition to fitting, BIGdippa incorporates analysis by a number of different DPPA methods, including the variance method and the Williamson-Hall method. Fourier methods are dealt exclusively by the GUI dippaFC.

For the FCC samples the average of the first five peaks are used and for the titanium samples the average of the first 10 peaks was used. The averaging is necessary because different peaks broaden by different amounts and in some instances some narrow when others broaden, particularly for Ti-6Al-4V. This effect is due to differences between samples (i.e. volume sampled and effect of texture) and effects from changes in dislocation population, and hence contrast factor, with applied strain.

\subsection{Williamson-Hall}

The Williamson-Hall method [5] is a popular method to determine size and strain values from the full-width or integral breadths of a diffraction pattern [27,42-47]. The advantage of the method is that only the fullwidth is needed so the quality of the peaks need not be as high as in some methods. The method works by fitting straight lines to plots of the full-width (or integral breadth) against the reciprocal of the lattice spacing; the gradient gives the micro-strain and the intercept the size. Different methods can be used depending on the separation of size and strain and the use of the contrast factor $[26,27]$.

The modified methods, which use the contrast factor and allow peaks from different families are:

$$
\begin{array}{ll}
\beta(g)=\frac{K_{S c}}{D}+f_{M} g \sqrt{\left(\rho \bar{C}_{h k l}\right)}+O\left(g^{2} \bar{C}_{h k l}\right) & \mathrm{mWH}-1 \\
\beta^{2}(g)=\frac{K_{S c}}{D^{2}}+f_{M}^{2} g^{2}\left(\rho \bar{C}_{h k l}\right)+O\left(g^{4} \overline{C_{h k l}^{2}}\right) & \mathrm{mWH}-2 \\
\beta(g)=\frac{K_{S c}}{D}+\sqrt{\rho} f_{M} g^{2} \bar{C}_{h k l}+O\left(g^{4} \overline{C_{h k l}^{2}}\right) & \mathrm{mWH}-3 \\
f_{M}^{2}=b^{2}\left\{a_{0} \ln (M+1)+b_{0} \ln ^{2}(M+1)+c_{0} \ln ^{3}(M+1)+d_{0} \ln ^{4}(M+1)\right\}
\end{array}
$$

where, $\rho$ is the dislocation density, $O(.$.$) gives information about the dislocation arrangement and for$ simplicity is ignored, $b(g)$ is the full-width of a particular peak with a reciprocal $d$-spacing of $g, K_{s c}$ is a constant $(=0.9)$, and $D$ is the crystal size. $f_{m}$ is a parameter that gives information about the dislocation structure, the above form of the formulae [48] is used with constants $a_{0}=-0.173, b_{0}=7.797, c_{0}=-4.818, d_{0}=$ 0.911. $\bar{C}_{h k l}$ is the contrast factor of dislocations and is used to account for why broadening does not change 
smoothly with $g$. We will use the approach of Ungar and colleagues to describe the contrast factor, although errors can be introduced by this use and this is discussed in a separate paper [23].

For the $\mathrm{mWH}$ results we use the first five diffraction peaks for FCC samples and the first 15 peaks for Ti-6Al$4 \mathrm{~V}$.

\subsection{Fourier-Methods}

The Warren-Averbach method [3] is the most used method to obtain details about the dislocation structure $[3,14,28,29]$, rather than just size and strain values. When the Warren-Averbach method is discussed it is normally with regard to one particular form, the 'log WA' form. But there is also a 'linear WA' version, which is just a different approximation to a Taylor series to separate size and strain terms. If the strain distribution is Gaussian, the log WA method is correct for all $g$ and $L$ values. The strain distribution won't be exactly Gaussian, and so in this form we are effectively assuming that the strain function is physically realistic and that $g$ and $L$ are small enough for the Taylor series approximation to be valid. In the linear WA form there is no strain distribution which would give the exact values for the Fourier coefficients [14]. van Berkum [14] believed that there are more systematic errors in the linear method, but less random errors compared to the log WA method. And although less used, some [49] have recommend it over the log form.

The Taylor series approximation to separate size and strain can be approximated in a different way [14] and is called the 'alternative method'. The main assumption made in this method is that strain gradients within domains are not important (known as the Stokes-Wilson approximation [41]) such that for large values of $L$ the displacement of the cells are large and random. The extreme case of this approximation is when there is a variation in intergranular strain in different grains but no distortion within the grains. The different methods that are investigated are:

- log-INDI-

- uses the log form of the Warren-Averbach method and Fourier coefficients with distinct values of $L$ are fitted separately

- $\log$-ALL-

$\circ$ uses the log form of the Warren-Averbach method and all the Fourier coefficients are fitted together

- lin-INDI-

0 uses the linear form of the Warren-Averbach method and Fourier coefficients with distinct values of $L$ are fitted separately

•individual-

- the same as log-INDI but peaks of the same family are used and the contrast factor is not used

- alternative- 
- uses the alternative method, peaks of the same family are used and the contrast factor is not used

- no-size-

- similar to log-INDI but it is assumed that there is no size broadening, and the strain is given by the full version of Groma [50]

More details on how the methods were implemented is provided in the Appendix and [38]. For the Fourier methods that use the contrast factor as a fitting parameter, the first five diffraction peaks for FCC samples and the first 15 peaks for titanium samples are used. And for the individual and alternative methods, the 111 and 222 peaks are used for FCC alloys and for Ti-6Al-4V the results are the average from the following families of peaks, (a) $10 \overline{1} 0,20 \overline{2} 0,30 \overline{3} 0$, (b) 0002, 0004, (c) $10 \overline{1} 1,20 \overline{2} 2$.

\section{Results}

\subsection{Full-Width}

The use of the full-width at half-maximum intensity of a peak (full-width) is the simplest and most widely used DPPA method. It is often used when other methods are not possible, either because the quality of the diffraction peak is low or there is a limited number of diffraction peaks available [23-25].

The change in the full-width with applied strain is shown in Figure 4a for the different alloys. As expected the full-width of all alloys increases with applied strain, as the grains become more distorted. A more useful way of interpreting the full-width changes is done by plotting the full-width against flow-stress (Figure $4 \mathrm{~b}$ ). This is because both the dislocation density [51,32,33] and the full-width [52] can be shown to be proportional to the flow stress. Consequently, if broadening is due only to dislocations, which do not change their arrangement or population, then the flow-stress should be proportional to the full-width. And a straight line fitted to the data would intercept the x-axis at the yield stress. The relationship observed is approximately linear for the alloys; although for Ni-200 at low stresses and SS-316 at high stresses the gradient increases slightly with stress. The value of stress when the data is extrapolated to the $x$-intercept is also close to the yield for all the alloys.

From these results, a good approximation for metals is that the full-width is directly proportional to the flow stress, with an intercept found from the yield stress. But the proportionality constant is material dependent and there can be problems at low stress values. 

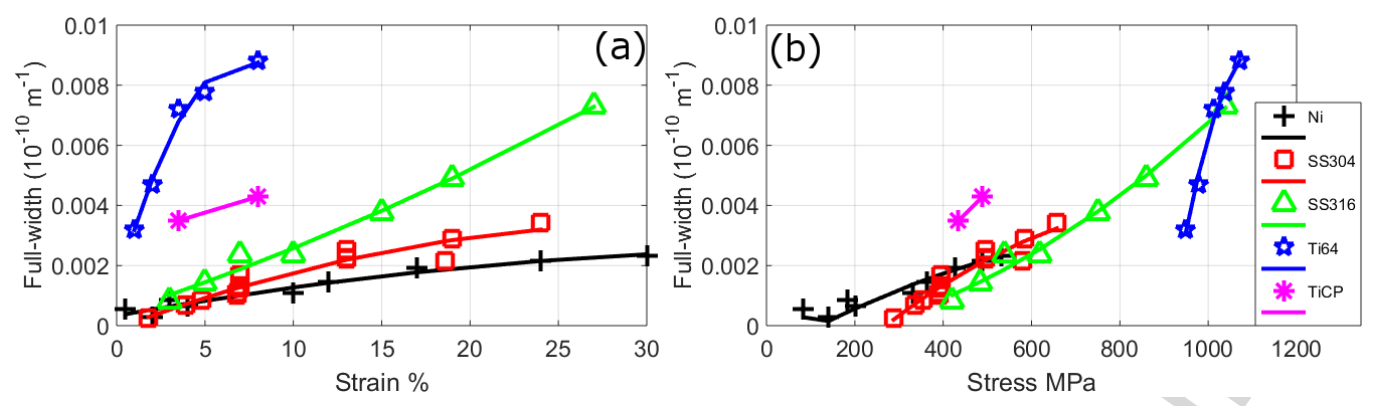

Figure 4, the change in full-width of the alloys plotted against the strain and flow stress. The lines represent a fit of the values to a $2^{\text {nd }}$ order polynomial.

\subsection{Dislocation density}

The dislocation density is an important property of a metal because it can be related to the flow stress of the alloy. And importantly it is a parameter that can be obtained by DPPA methods. In this part the dislocation density values $(\rho)$ obtained from the different methods are presented. The results are compared with values obtained on similar alloys by TEM using the Taylor equation (Equation 2). This equation relates the dislocation density $(\rho)$ to the flow stress $(\sigma)$, by a constant of proportionality $\alpha^{\prime}$. This relationship has been observed in numerous metals and is found to be independent of the dislocation structure or the crystal structure of the metal [32-34].

$$
\sigma=\sigma_{0}+\alpha^{\prime} \mathrm{Gb} \sqrt{\rho}
$$

where, $G$ is the shear modulus and $b$ the magnitude of the Burgers vector of dislocations, $\sigma_{0}$ is the friction stress, or the yield stress of an annealed sample, and $\alpha^{\prime}$ is a constant that is found to change depending on the material studied. From the TEM results of Lee and Lin [35] a value of $\alpha^{\prime}=0.48$ was found for SS-316. For comparison with the nickel results, we will use two different results as reference; a value of $\alpha^{\prime}=1$ was found by Gubicza et al. [7] using DPPA on nickel, and for copper (a metal with a high SFE like nickel) Mecking and Kocks [34] obtained a value of $\alpha^{\prime}=0.5$ from TEM and $\alpha^{\prime}=1$ using an etch pit technique. For titanium it has been found $[33,22]$ that the value of $\alpha^{\prime}$ is independent of interstitial content, temperature, dislocation structure or grain size and can vary between $\alpha^{\prime}=0.34$ and $\alpha^{\prime}=0.9$.

Figure 5 shows the change in the dislocation density found from different Williamson-Hall methods, and in Figure 6 using different Fourier methods. When calculating the values using the Williamson-Hall method we have assumed a constant value of the dipole character of $M=0.23$ for all three methods; this was chosen so that the DPPA results are close to the expected changes of dislocation density with stress. The usefulness of assuming a constant $\mathrm{M}$ with the method has shown some success [53]. The DPPA data was fitted to Equation 2 to find the proportionality constant $\alpha^{\prime}$ and the friction stress $\sigma_{0}$. The ratio of these values with the expected values, using values of $\alpha^{\prime}, G$ and $B$ from other studies, are shown in Table 2. For Ti-6Al-4V it is assumed that all dislocations are $<\mathrm{a}>$ dislocations because they are expected to be the dominant dislocation [17]. 
In general, the dislocation density values change linearly with stress. But there are different values of $\alpha^{\prime}$ and $\sigma_{0}$ for the different metals and methods. Because we have set the value of $\mathrm{M}$, used in the mWH methods, the change in dislocation density with stress are closest for the $\mathrm{mWH}$ methods. It is worth noting that the difference in the gradient of the full-width against stress between Ti-6Al-4V and the FCC alloys (in Figure 4) is now less evident because of the differences in the constants of equation 1 and 2 (particularly for the $\mathrm{mWH}$ methods).

Table 2, the change in parameters $\alpha$ ' and $\sigma_{\omega}$ found by fitting DPPA data to Equation 2, are used to show how the DPPA dislocation density values changes with flow stress for different methods. The ratio of DPPA $\alpha^{\prime}$ values to the expected values $\alpha^{\prime}$ from TEM data (details in body of text) is shown. Along with the ratios of the DPPA $\sigma_{0}$ values to the $0.1 \%$ yield strength of the different alloys by the Yield ratio parameter. Descriptions of the Williamson-Hall methods ( $m W H-1,2,3)$ can be found in Equation 1 and Section 3.1, and the Fourier methods in Section 3.2.

\begin{tabular}{|c|c|c|c|c|c|c|c|c|c|c|}
\hline \multicolumn{2}{|l|}{ Method } & $\begin{array}{c}m W H- \\
1\end{array}$ & $\begin{array}{c}m W H- \\
2\end{array}$ & $\begin{array}{c}m W H- \\
3\end{array}$ & $\begin{array}{l}\text { log- } \\
\text { INDI }\end{array}$ & $\begin{array}{l}\text { lin- } \\
\text { INDI }\end{array}$ & $\begin{array}{l}\text { log- } \\
A L L\end{array}$ & $\begin{array}{c}\text { altern } \\
\text { ative }\end{array}$ & indiv. & no-size \\
\hline \multirow{3}{*}{ Nickel } & $\alpha^{\prime}$ ratio & 1.01 & 0.90 & 0.60 & 2.17 & 2.28 & 0.48 & 3.17 & 1.87 & 1.09 \\
\hline & $\begin{array}{l}\text { Yield } \\
\text { ratio }\end{array}$ & 2.11 & 1.78 & 1.85 & -7.08 & -7.38 & -6.92 & -13.85 & -5.85 & -4.29 \\
\hline & \multicolumn{2}{|c|}{ Expected $\alpha^{\prime}$} & 0.75 & Yield & 65 & & & & & \\
\hline \multirow{3}{*}{ SS304 } & $\alpha^{\prime}$ ratio & 1.07 & 0.86 & 0.47 & 1.46 & 1.27 & 1.04 & 2.31 & 1.73 & 0.67 \\
\hline & $\begin{array}{l}\text { Yield } \\
\text { ratio }\end{array}$ & 1.43 & 1.41 & 1.46 & 0.34 & 0.57 & 0.63 & -0.08 & -0.09 & 1.04 \\
\hline & \multicolumn{2}{|c|}{ Expected $\alpha^{\prime}$} & 0.48 & Yield & 192 & & & & & \\
\hline \multirow{3}{*}{ SS316 } & $\alpha^{\prime}$ ratio & 1.13 & 1.12 & 1.30 & 3.25 & 6.00 & 1.60 & 9.38 & 0.88 & 0.65 \\
\hline & $\begin{array}{l}\text { Yield } \\
\text { ratio }\end{array}$ & 1.13 & 1.12 & 1.30 & -1.70 & -3.85 & -0.77 & -3.85 & 0.96 & 0.68 \\
\hline & \multicolumn{2}{|c|}{ Expected $\alpha^{\prime}$} & 0.48 & Yield & 312 & & & & & \\
\hline \multirow{3}{*}{$\begin{array}{c}T i-6 A L- \\
4 V\end{array}$} & $\alpha^{\prime}$ ratio & 1.00 & 0.98 & 1.37 & 0.79 & 2.18 & 1.23 & 14.03 & 0.65 & 0.82 \\
\hline & $\begin{array}{l}\text { Yield } \\
\text { ratio }\end{array}$ & 0.88 & 0.88 & 0.88 & 0.92 & 0.65 & 0.87 & -0.66 & 0.93 & 0.81 \\
\hline & \multicolumn{2}{|c|}{ Expected $\alpha^{\prime}$} & 0.62 & Yield & 943 & & & & & \\
\hline \multirow[b]{2}{*}{ Average } & $\alpha^{\prime}$ ratio & 1.05 & 0.97 & 0.94 & 1.92 & 2.93 & 1.09 & 7.22 & 1.28 & 0.81 \\
\hline & $\begin{array}{l}\text { Yield } \\
\text { ratio }\end{array}$ & 1.39 & 1.30 & 1.37 & -1.88 & -2.50 & -1.55 & -4.61 & -1.01 & -0.44 \\
\hline
\end{tabular}

A significant difference is found in the values of $\sigma_{0}$ obtained by the Williamson-Hall and Fourier methods. For the Williamson-Hall methods the fitted lines intersect the $\mathrm{x}$-axis at values close to the yield, albeit at slightly higher values for Ni-200 and lower values for Ti-6Al-4V. But for the Fourier methods the lines intersect the $\mathrm{x}-$ axis at much lower values, and in many cases the value is negative. This behaviour is particularly noticeable in Ni-200 and SS-316 alloys, although it does occur to a lesser extent for the others. It is largest in the alternative and lin-INDI methods, and lowest for the no-size method. The behaviour has not to the author's knowledge been observed in either DPPA or TEM measurements. Hence, there are a number of possible causes for the behaviour, the most probable of these are the Fourier results contain systematic errors. These 
could be a result of (a) the separation of instrumental broadening, (b) the separation of size and strain contributions, or (c) the separation of dislocation density and arrangement. Because the effect is reduced when we ignore size broadening (no-size method) or when the instrumental broadening is lower (Ti-6Al-4V samples), it is probable that both (a) and (b) have an influence on this behaviour. The values of $\alpha^{\prime}$ found when this behaviour is observed are much larger than the expected values. However, the dislocation density values at the higher stresses are close to those expected. This would suggest there is a systematic error causing higher dislocation densities at lower stresses than expected.
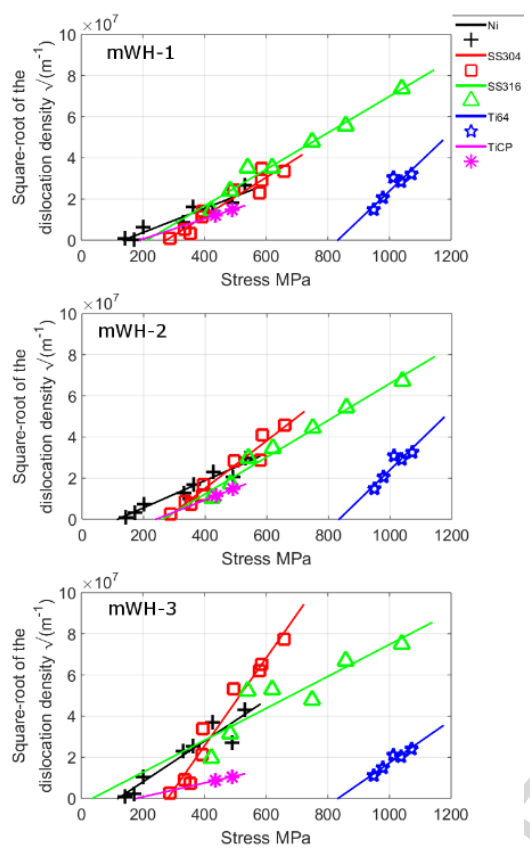

Figure 5, the change in the square-root of the dislocation density found by different Williamson-Hall methods with stress. The lines represent a fit of the values to a straight line. 

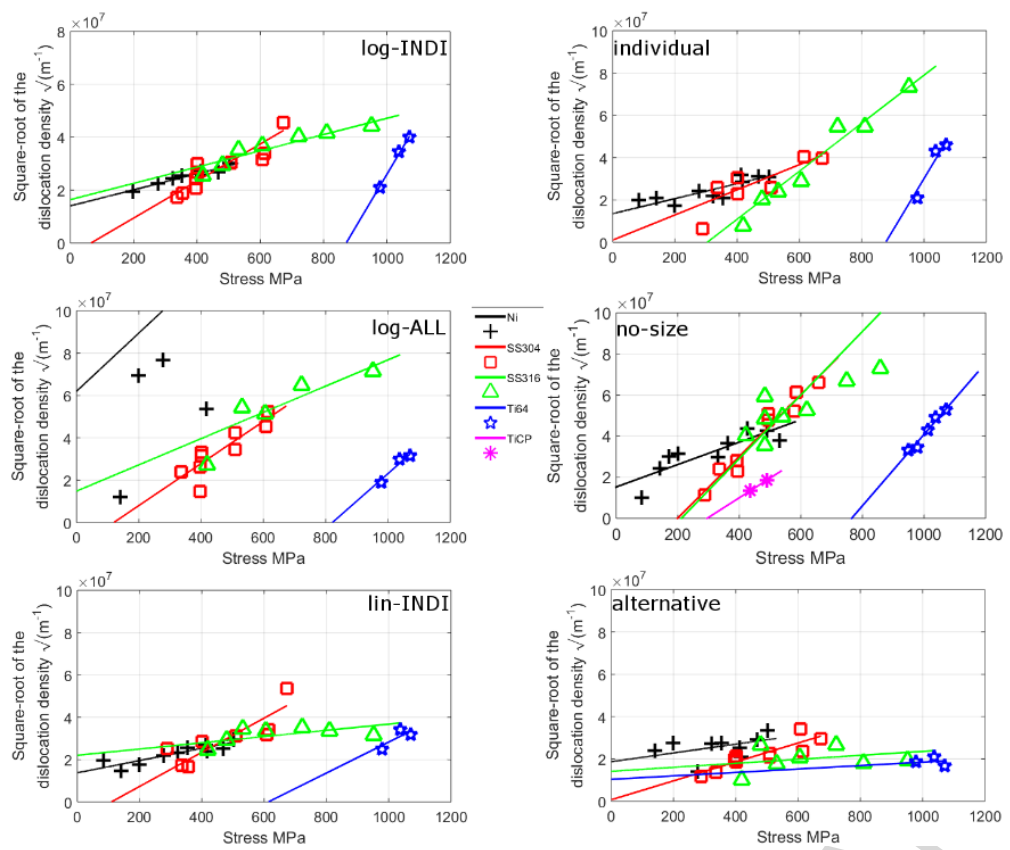

Figure 6, the change in the square-root of the dislocation density found by different Fourier methods with stress. The lines represent a fit of the values to a straight line.

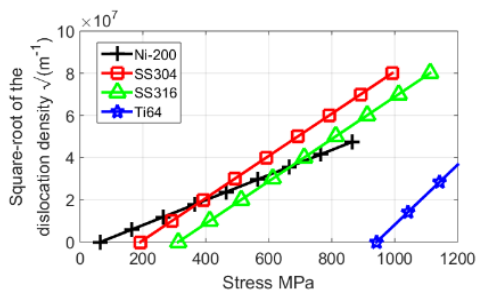

Figure 7, the expected change in the square-root of the dislocation density with stress using Equation 2, with the $0.1 \%$ yield stress as $\sigma_{0}$ and the values of $\alpha^{\prime}$ from TEM results of other researchers.

There are a range of $\alpha^{\prime}$ values (and dislocation densities at a particular stress) for the different WA methods and no obvious trend to suggest which would give the highest or lowest values. These differences are significant and are a reflection of both random and systematic errors that result from applying the different methods. The alternative method provides the largest values of $\alpha^{\prime}$ of all the methods and in most cases these values are larger than expected. These higher values of $\alpha^{\prime}$ correspond to lower values of the dislocation density for a given stress. This shows that even though the alternative method gives a smaller contribution to size broadening, for the low SFE metals, this does not equate to higher values of the dislocation density. The main cause for the larger value of $\alpha^{\prime}$ for the alternative method, is probably due to the different size and strain separation used. The no-size approach provides the lowest value of $\alpha^{\prime}$, corresponding to higher values of the dislocation density for a given stress. Which may suggest that a part of the broadening is due to size broadening.

\subsection{Dislocation Arrangement}

The strain field around a group of dislocations is different than the strain field of individual dislocations. The reason for this is that the total strain field of a group of dislocations is found from the interaction of the 
strain fields of the individual dislocations. This means that for the same dislocation density the broadening will vary depending on how the dislocations are arranged. In DPPA methods, this arrangement is quantified by a variable called the dipole character, or $M[4,54]$. The problem with this parameter is that it is not possible to measure by other characterisation methods, meaning that in most cases the dipole character is only used as a fitting parameter. However, in theory the value should be related to the dislocation structure, and determination of the variable is important to avoid introducing systematic errors or when trying to obtain dislocation density values from methods that only give micro-strain (e.g. Williamson-Hall methods).

In Figure 8 are the values of the dipole character for selected Warren-Averbach methods. For log-ALL WA method there is a greater scatter in $M$ than for the size or dislocation density values, which makes its behaviour harder to quantify. The magnitudes of $M$ are highest for the alternative method, $M \sim 7$ for Ti-6Al$4 \mathrm{~V}$, then the log methods and then the lin methods, for lin-INDI using Ti-6AI-4V gives a value of $M^{\sim} 1$. There is a general trend for each method and alloy: the value of $M$ increases with applied strain and then saturates. The general behaviour of $M$, increasing and then levelling off, is in agreement with the expectations and the results of Zehetbauer et al. [55] on copper polycrystals. They observed an increase in $M$ up to a strain of around 0.2 , followed by a plateau. They explained the initial increase in $M$ as being due to regions with low and high dislocation density developing with increasing applied strain. The plateau was explained as being due a transition in the type of dislocations present from those with a higher value of $M$, which are dominant at low strains, to those with a lower value of $M$, which become dominant at higher strains. A lower $M$ value is obtained when dislocations organise into dislocation structures because they screen the strain field of each other. Hence, for all metals there may be two competing influences on the value of $M$. An increase with applied strain due to increases in dislocation density and a reduction due to the formation of a more organised dislocation structure. Although this behaviour was explained for a high SFE metal, this same change in arrangement does occur for metals of lower SFE, it is just slower. For example, in stainless steel $[56,57]$ dislocations are mainly organized as tangles at low stresses and with increased stress these change to dislocation cells. Of the alloys nickel has the lowest value of $M$, whereas the values for stainless steel alloys and Ti-6Al-4V are comparable. These differences are consistent with the differences in SFE of the alloys and the description of $M$ being lower when dislocations become more organised. 

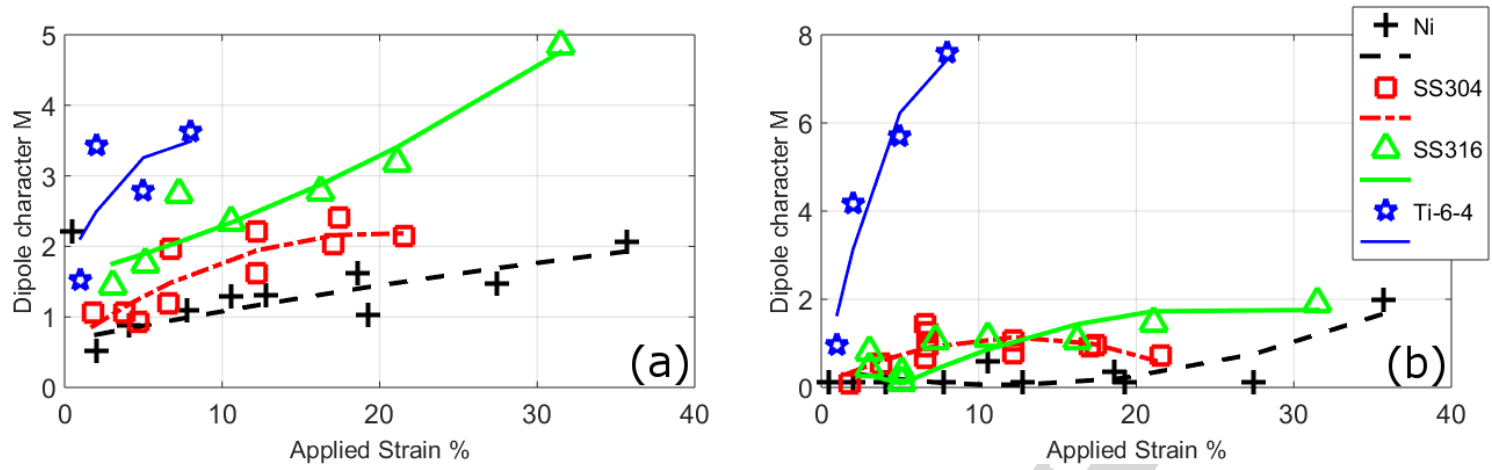

Figure 8, results of the dipole character $M$, for selected Fourier methods applied to the different alloys. The results are for the WA $\begin{array}{lllll}\log -I N D I & (a) & \text { and } & & \end{array}$ (b)

The dislocation density and dipole character are closely related and it can be difficult to separate them. An increase in both causes an increase in broadening, so if the value of $M$ found by DPPA is lower than its actual value we would obtain a higher dislocation density. Hence, the changes observed for the dipole character (an increase in $M$ at lower strains) may not be real changes of the microstructure but could be artefacts from the fit. This may explain (at least partly) the reason for the systematic differences between the dislocation density results and the Taylor equations at low applied strains, found in the previous section for the Fourier methods. But if these changes in $M$ are correct this would mean the dislocation density values found from the Williamson-Hall methods, where $M$ is assumed constant, would not be correct.

There is some justification that the values and trends seen for the dipole character represent the metals, because the behaviour of the dipole character follows a trend found and explained by other researchers, and the difference in its magnitude between steel and nickel samples is expected given the differences in the deformed microstructure. However, there are still some un-answered questions. Why does the value of $M$ change depending on the method used? What exact values and trends are expected and how can the value be compared to TEM results? Why does a constant value of $\mathrm{M}$ in the Williamson-Hall methods give the change in dislocation density expected? Without an understanding of these questions, the dipole character becomes just a fitting parameter that may cause systematic errors.

\subsection{Size}

When there is no micro-strain present in a sample, the use of DPPA methods have been effective in determining the size of crystals $[1,11,12]$. However, when both size and strain broadening are present there is an ambiguity over what the size from DPPA actually represents [11,14].

The size in DPPA is the length of a region that diffracts incoherently (or diffracts independently) with respect to all other regions [3]. A region diffracts incoherently with another region when it is separated by a grain boundary, because of the difference in orientation. However, when the subgrain size is measured in metals with high SFE (and develop a cell structure), there is often a discrepancy between TEM (or optical, or EBSD) size values and those found using DPPA $[1,11,14]$. It has been suggested that the discrepancy is due either to 
a difference in the size distributions measured by the methods [11]. Or that the walls of dislocation cells cause a break in coherency, even though they have no misorientation across them [58]. However, van Berkum et al. [14] argued that the discrepancy was caused by limitations of the mathematics involved in separating size and strain values, for example caused by the Taylor series approximation in the WarrenAverbach method. Their idea of what would cause size broadening is different. They believed that only high angle boundaries or small angle boundaries with large local strain concentrations could cause a break of coherency.

In order to understand the meaning of size in DPPA we first consider the size values obtained for Ni-200. Nickel, due to its high SFE, develops a dislocation cell structure more readily than the others. Hence, it is the only alloy that has a DPPA size that can be compared with other measurements. When a cell structure develops, the dislocation cell size (D) has been found to be almost inversely proportional to the flow stress $[18,36,59]$.

$D=\Omega b\left(\frac{G}{\sigma}\right)^{m}$

where, $\Omega$ is a constant found to be 14 for nickel [59], $m$ a constant found to be 0.98 for nickel [59], $G$ is the shear modulus and $b$ the magnitude of the Burgers vector.

The change of the crystal size of nickel for selected methods is shown in Figure 9, and the fits of Equation 3 to the crystal sizes from each method is shown in Table 3. The crystal size falls for all methods with increasing flow stress. But there are significant differences in the crystal values obtained, and the values of $m$ and $\Omega$. The value of $m$ varies between 0.5 to 1.3 for the methods, with an average that is close to the expected value of 1 from TEM [36,59]. We can compare the absolute values of different methods by fitting to the size values with a constant value of $m$, this is shown in the right of Table 3 . In all cases the DPPA results are smaller than the TEM results. The ratio of sizes of TEM [59] to DPPA varies from around 1:2 (for $\mathrm{mWH}-3$ ) to 1:10 (for Variance Voigt), with an average ratio of 1:7. These values are consistent with comparison between TEM and DPPA; Ungar [58] compiled data on plastically deformed metals and showed that the ratio of sizes from TEM to DPPA varied from around 3 times at a crystal size of $750 \AA$, to 7 times at $5000 \AA$.

The two methods with the highest crystallite size values are the modified Williamson-Hall and WarrenAverbach log-ALL methods. This may be due to the slightly different interpretations of the crystal size than for other methods. The crystallite size from Williamson-Hall methods represents the volume weighted crystal size, whereas the other methods give the area weighted crystal size. And as found by Ungar [11] the volume-weighted size should always be more than or equal to the area-weighted value. A possible reason for the difference in the log-ALL method is that instead of assuming a set size distribution, we have used a lognormal distribution and fitted the size to the mean and the standard deviation (and the other methods we only fit to the mean). 

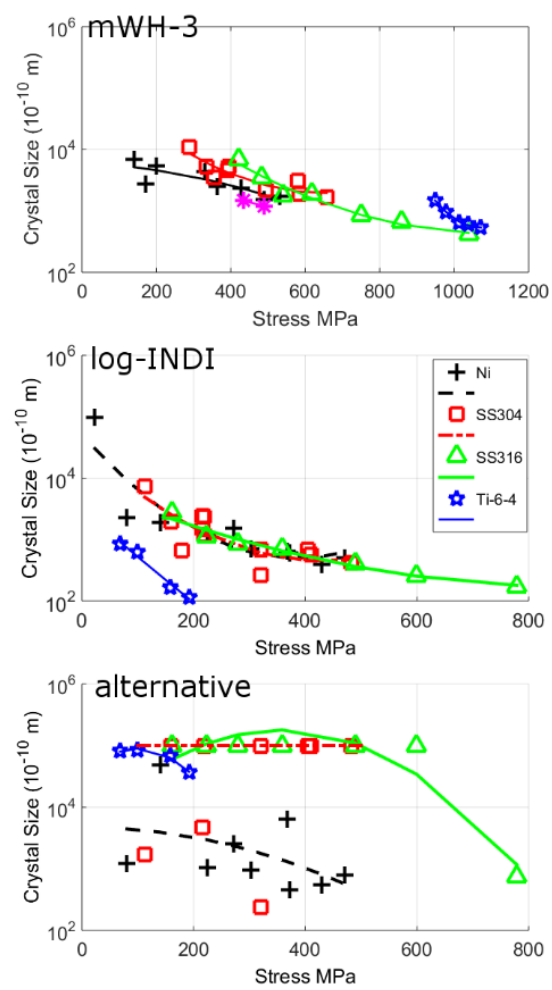

Figure 9, the change in crystal size (in $\AA$ ), plotted against work-hardening stress. The results are for the mWH-3, WA log-INDI and alternative methods. The lines represent a fit of the values to $a 2^{\text {nd }}$ order polynomial.

In metals with lower stacking fault energies, such as stainless steel and Ti-6Al-4V, the dislocation structures that develop are markedly different from nickel (Figure 10)[18,22,60-62]. In these metals the microstructure is dominated by dislocation tangles and dislocation walls, which lie along the dominant slip planes $[56,57]$ rather than the subgrain and cell structure found in nickel. Dislocation cell structures can develop but are not as well defined as those in nickel. Given this difference it may be expected that the crystallite size values from DPPA, of stainless steel and Ti-6Al-4V, would be larger than those found for nickel. But this is not what is found (Figure 9). Instead, the crystallite size values are comparable for both groups of alloys. The exception to this is the alternative method where crystal size broadening is only significant for $\mathrm{Ni}-200$. This is consistent with the work by the methods developers [14], who showed that for a deformed metal with a relatively large crystal size the alternative method gave size broadening comparable with the size of the crystals, but the Warren-Averbach method gave considerably smaller crystal sizes. They attributed the lower crystal size for the Warren-Averbach method as being an artefact from incorrect size and strain separation.

The traditional view of crystal size is that it is the size of a region that diffracts incoherently with all other parts of a crystal, because it has a different orientation to neighbouring regions. However, the results of this chapter show that this description is not correct. In the metals studied those that are not expected to consist of small crystals misorientated from each other (Ti-6Al-4V and stainless steel) have DPPA crystal size values of similar magnitude to those that do (nickel). Hence, the crystal size can be thought of as either being due to an error from size and strain separation or the size of an undistorted part of a crystal. This undistorted 
size component can be thought of as being caused by dislocations, that do not necessarily have a misorientation across them. The problem with this description of the crystal size is that it has little use in characterising a metal. The exception to this obscure definition of crystal size is found with the alternative method, which gives a falling crystal size value for nickel, but no size broadening for the low SFE metals. Hence, the alternative method is the only one that gives a description of crystal size that can be verified.

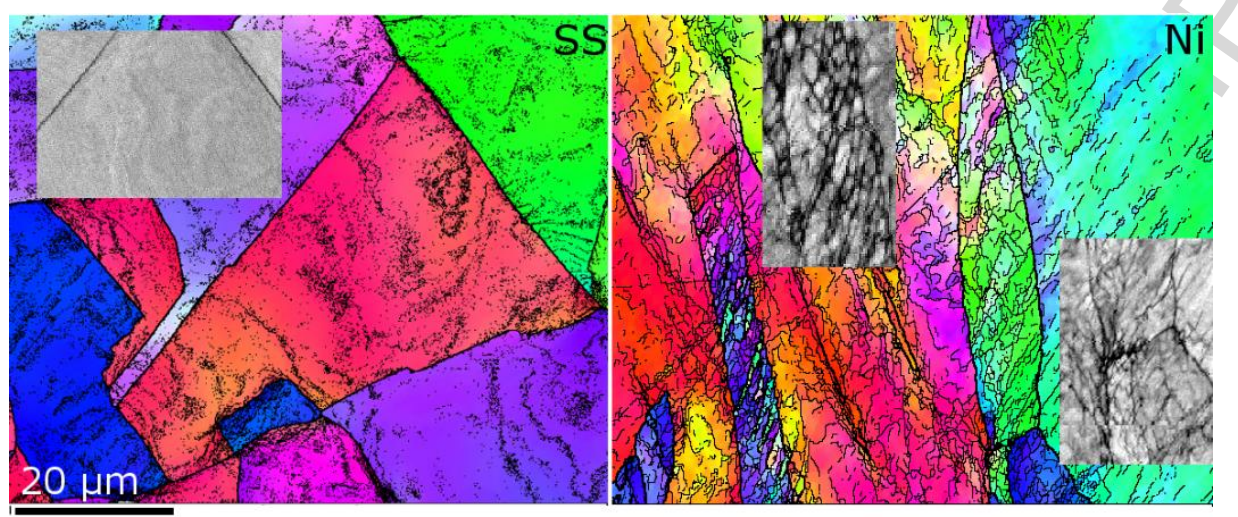

Figure 10, The microstructure of SS-316 (left, SS) and Nickel-200 (right, Ni) after deformation to 10\% applied strain from EBSD. The main images are plots of orientation colours (IPF) with boundaries of $0.7^{\circ}$ misorientation. The smaller images are magnified plots of selected regions using the band-contrast and display the distortion around dislocations. The images show a subgrain structure has developed in Ni-200 but not in SS-316.

Table 3, The change in parameters $\Omega$ and $m$ (found from fitting data to Equation 3), used to show how the crystal size changes with flow stress, for different DPPA methods. These parameters are determined allowing both to change and only allowing $K$ to change. The expected values are compared with TEM data on aluminium [36]* and nickel-200 [59]**.

\begin{tabular}{l|llll} 
Method & $\mathbf{m}$ & $\boldsymbol{\Omega}$ & $\mathbf{m}$ & $\boldsymbol{\Omega}$ \\
\hline log-INDI & 1.33 & 0.25 & 0.96 & 1.8 \\
log-ALL & 0.67 & 20.6 & 0.96 & 4.2 \\
lin-INDI & 1.28 & 0.3 & 0.96 & 1.8 \\
individual & 0.85 & 3.4 & 0.96 & 1.8 \\
ALT & 0.59 & 14.9 & 0.96 & 2.0 \\
mWH-3 & 0.72 & 23 & 0.96 & 6.2 \\
\cline { 1 - 1 } Expected- Al* & 0.95 & 25 & & \\
Expected- Ni** & 0.98 & 13.7 & &
\end{tabular}

\subsection{Errors and Scatter of DPPA results}

There are two aspects to whether a particular method is useable, one is that the method gives the correct absolute values; but it is also important to know the repeatability and reliability of a method. Figure 5 and Figure 6 can be used to give an indication of the random errors or scatter in the DPPA results. We can see that for the Williamson-Hall methods the data is relatively close to the line fits, with the scatter being the largest for $\mathrm{mWH}-3$. For the Fourier methods the scatter is in general higher than for the Williamson-Hall 
methods. With the scatter being largest for the log-ALL approach, but the scatter is also slightly higher for the INDI-type approaches.

The choice of the range of the Fourier coefficients used when fitting using the log-ALL approach is an important parameter that needs consideration. The effect of the fitting range was investigated with a Ti-6Al$4 \mathrm{~V}$ sample (where instrumental broadening is lowest) and is shown in Figure 11. When the range selected is sufficiently large, such that the Fourier coefficients are less than around 0.1 , the results are almost constant. However, for ranges smaller than this the values vary significantly: an increase in the crystal size and dislocation density and a fall in the dipole character. So this would suggest that we should make the fitting range sufficiently large; however, this is not always possible. For the laboratory x-ray samples in particular, the Fourier coefficients often became unreliable at a certain values of $L$ (Figure 12) which can equate to values of the Fourier coefficients that are larger than 0.1 . This is particularly the case at low amounts of broadening and is probably due to deconvolution and instrumental effects. The Warren-Averbach method is only valid for a given strain distribution and for small values of $L$, and as $L$ increases so will the error of using this approach $[3,14]$. Hence, this is another potential source of errors when using the log-ALL approach. These two effects may explain the difficulty sometimes observed in the FCC samples in separating dislocation density and dipole character, and the increased scatter.

In the INDI approaches we are able to get around this problem, found in the log-ALL approach, by fitting the Fourier coefficients at particular values of $L$. The range chosen was approximately between $L$ values of $100 \AA$ to $300 \AA \hat{A}$. The region was chosen because it is least affected by background (low L), or instrumental effects and limitations of the separation method (high $L$ ). The dislocation density was separated from the dipole character, $M$, by fitting straight lines to the $\log$ of the strain coefficients $\left(\ln A_{D}\right)$ against the $\log$ of the Fourier length $(L)$, which is called the Krivoglaz-Wilkens plot [1]. The dislocation density $(\rho)$ is found from the gradient, the dipole character $(M)$ is found from the intercept.

$\frac{\ln A_{D}(L)}{L^{2}}=\rho \bar{C} B \ln \left(R_{e}\right)-\rho \bar{C} B \ln (L), \quad \mathrm{B}=\pi \mathrm{b}^{2} / 2, M=R_{e} \sqrt{\rho}$

In many cases the Krivoglaz-Wilkens (K-W) plot (Figure 13a) displays a defined linear region. But in some cases the behaviour can be curved. The K-W plot in Figure 13b found using the lin-INDI approach is curved and so the dislocation density and $M$ values vary significantly depending on which part is fitted; if we fit to the lower $L$ range the dislocation density is $60 \%$ higher and $M 24 \%$ lower than when fit to the high $L$ values. This curvature was often observed for the lin-INDI approach, but was much more linear for the log-INDI approach. Hence, the choice of the L-range to determine the dislocation density values in the INDI approaches has the potential to cause significant errors. 

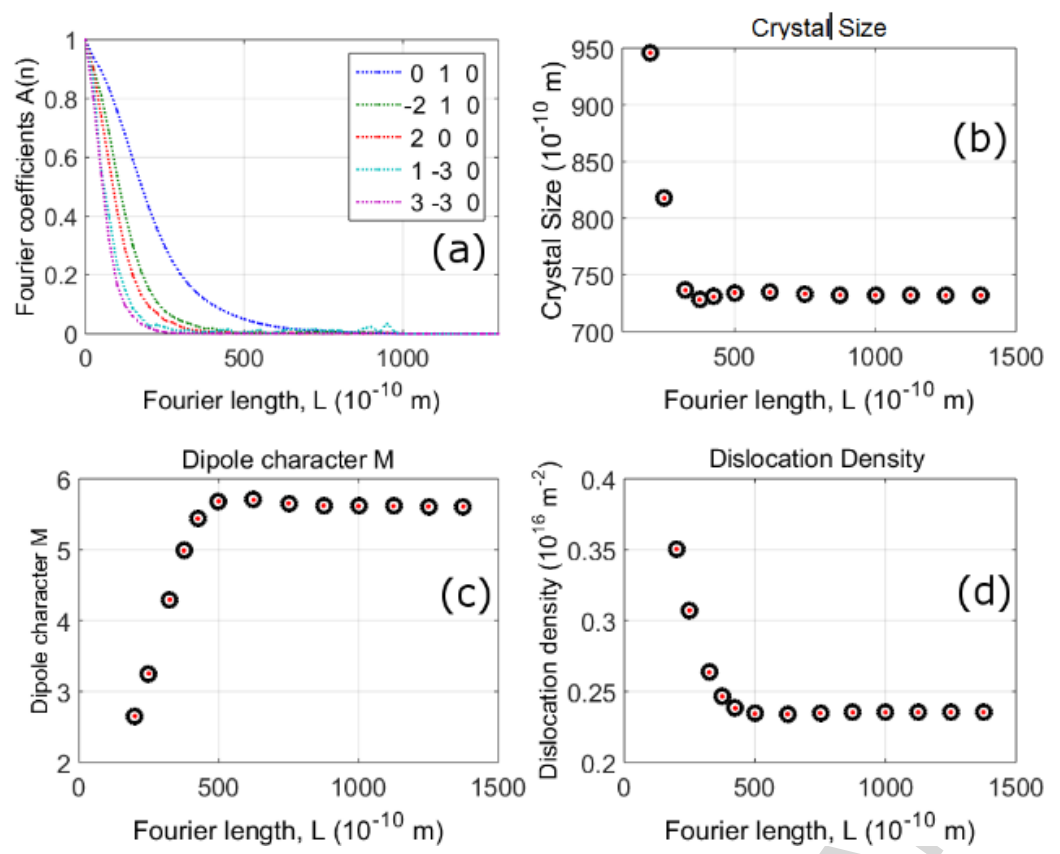

Figure 11, an example of the change in the parameters obtained from a WA log-ALL analysis when the fitting range is changed. This is done for Ti-6Al-4V in the axial direction after 5\% applied strain.

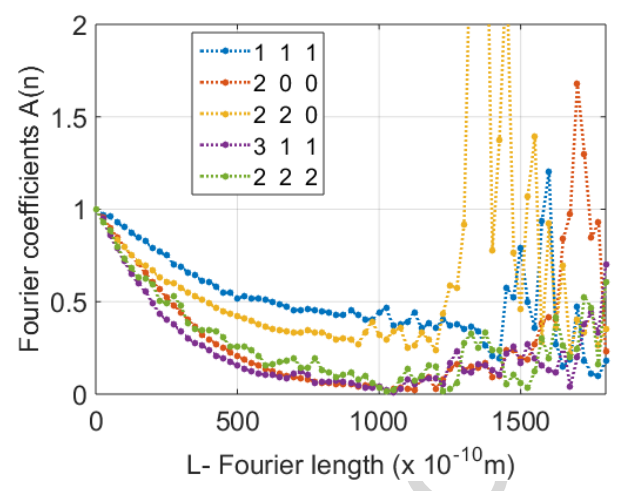

Figure 12, the Fourier coefficients of SS-316 at 5\% applied strain after removing instrumental broadening.
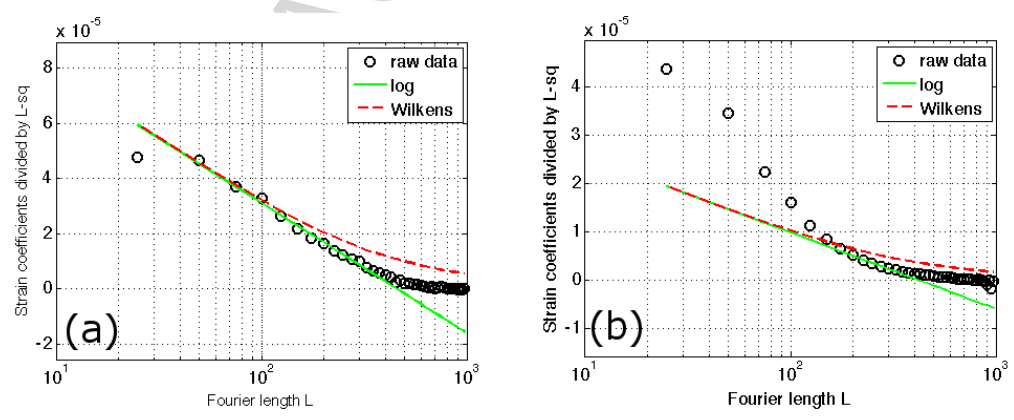

Figure 13, example Krivoglaz-Wilkens (K-W) plots for Ti-6Al-4V in the axial direction after $5 \%$ applied strain. $K$-W plots of the log of the Fourier length against the square of the strain coefficients divided by $L^{2}$. (a) log-INDI, (b) lin-INDI. For the lin-INDI sample because of the curvature of the values, a fit to the left of the curve (low L) would give dislocation density $~ 60 \%$ higher and an $M$ value $24 \%$ lower than if fitted to the right (high L). 


\section{Conclusions}

The use of diffraction peak profile analysis (DPPA) methods to characterise plastically deformed alloys was investigated. We considered the usefulness of different Williamson-Hall and Fourier methods, to obtain details of the microstructure of FCC and HCP alloys strained by different amounts.

In general, the dislocation density values found by both Williamson-Hall and Warren-Averbach methods are close to those expected from TEM results of similar metals. However, there is considerable spread in the changes of dislocation density from different methods and alloys. And in contrast, the values found by the alternative method are lower than expected. The Fourier results show a systematic error from the expected values at low amounts of plastic deformation, which is not observed in the Williamson-Hall results. This systematic difference causes higher than expected dislocation density values at values of low flow stresses and strains, and is thought to be due three factors: (1) separation of instrumental broadening, (2) separation of size and strain broadening, and (3) separation of dislocation density and arrangement.

Potential problems were identified when using the Fourier methods that could lead to errors. The main problem with fitting all the coefficients together is the choice of what range of Fourier lengths to fit to, which is a particularly problem when the instrumental is broadening is high relative to the samples real broadening. When using Fourier methods that fit the Fourier coefficients individually there can be issues caused by a curvature in the Krivoglaz-Wilkens plots which adds an uncertainty to the results.

The advantage of the Williamson-Hall methods is that some of these problems encountered by the Fourier methods were not found. In this method we have assumed dislocation arrangement (given by the dipole character) is constant with applied strain and is given by the same value of the dipole character for all metals. But from the Fourier methods the dipole character was found to change with applied strain. If the value is changing, then this would change the dislocation density values found by the Williamson-Hall method. More research is therefore needed to interpret this value in order to help quantify DPPA results.

The usefulness of DPPA methods to determine details of the dislocation cell size of Ni-200 was investigated. The size values from the DPPA methods changes in approximately the expected manner. The results obtained were found to be smaller than TEM results from similar alloys with a ratio of sizes of consistent with comparison between TEM and DPPA [58]. However, the same change in size was obtained for the alloys with higher stacking fault energies that don't develop a cell structure. Which leads to a conclusion that the size from DPPA is either an artefact from size and strain separation or some measure of the separation of dislocations. The only method that was able to distinguish alloys with a defined cell structure, from those without, was the alternative method. In this method Ni-200 showed size broadening but the size broadening was minimal for the other alloys. Hence, the alternative method is the best method to understand changes in the size of the dislocation structure of plastically deformed metals. 


\section{Acknowledgements}

The authors would like to thank the following people for their help; Alex Evans and the team at ESRF for their help with the beamline experiment; Levante Balogh for help at the ESRF; Tamas Ungar for discussions on peak profile methods; Judith Shackleton for help with laboratory X-ray measurements; And Alex Forsey for suggestions on code improvements. The authors are grateful to funding by DTA account, through EPSRC.

\section{REFERENCES}

[1] R. Kuẑel, Zeitschrift Fur Krist. 222 (2007) 136-149.

[2] A. Borbely, T. Ungar, Comptes Rendus Phys. 13 (2012) 293-306.

[3] B.E. Warren, X-Ray Diffraction, Addison-Wesley Publishing Co., Reading, 1969.

[4] M.A. Krivoglaz, X-ray and Neutron Diffraction in Nonideal Crystals, Springer-Verlag, Berlin, 1996.

[5] G.K. Williamson, W.H. Hall, Acta Metall. 1 (1953) 22-31.

[6] K. Venkateswarlu, M. Sandhyarani, T.A. Nellaippan, N. Rameshbabu, Procedia Mater. Sci. 5 (2014) 212221.

[7] J. Gubicza, N.Q. Chinh, G. Krallics, I. Schiller, T. Ungar, Curr. Appl. Phys. 6 (2006) 194-199.

[8] P.S. Roodposhti, A. Sarkar, K.L. Murty, R.O. Scattergood, Metallogr. Microstruct. Anal. 4 (2015) 337343.

[9] M.A. Vicente Alvarez, J.R. Santisteban, P. Vizcaíno, G. Ribárik, T. Ungar, Acta Mater. 117 (2016) 1-12.

[10] A. Wauthier-Monnin, T. Chauveau, O. Castelnau, H. Réglé, B. Bacroix, Mater. Charact. 104 (2015) 3141.

[11] T. Ungár, Adv. Eng. Mater. 5 (2003) 323-329.

[12] D. Balzar, N. Audebrand, M.R. Daymond, A. Fitch, A. Hewat, J.I. Langford, A. Le Bail, D. Louer, O. Masson, C.N. McCowan, N.C. Popa, P.W. Stephens, B.H. Toby, J. Appl. Crystallogr. 37 (2004) 911-924.

[13] P. Bindu, S. Thomas, J. Theor. Appl. Phys. 8 (2014) 123-134.

[14] J.G.M. van Berkum, A.C. Vermeulen, R. Delhez, T.H. de Keijser, E.J. Mittemeijer, J. Appl. Crystallogr. 27 (1994) 345-357.

[15] N.S. Gonçalves, J.A. Carvalho, Z.M. Lima, J.M. Sasaki, Mater. Lett. 72 (2012) 36-38.

[16] H.M. Ledbetter, Phys. Status Solidi. A85 (1984) 89-96.

[17] G. Lütjering, J. Williams, Titanium: Engineering Materials and Processes, Springer, Manchester, UK, 2007.

[18] E.H. Lee, T.S. Byun, J.D. Hunn, M.H. Yoo, K. Farrell, L.K. Mansur, Acta Mater. 49 (2001) 3269-3276.

[19] J. Luo, P. Ye, M.Q. Li, L.Y. Liu, Mater. Des. 88 (2015) 32-40.

[20] B. Ma, C. Li, J. Zheng, Y. Song, Y. Han, Mater. Des. 92 (2016) 313-321. 
[21] K. Li, X. song Fu, R. dong Li, W. long Zhou, Z. qiang Li, Mater. Des. 86 (2015) 761-764.

[22] C.P. Biswas, Strain Hardening of Titanium by severe plastic deformation, PhD thesis, MIT, 1973.

[23] T.H. Simm, P.J. Withers, J. Quinta Da Fonseca, J. Appl. Crystallogr. 47 (2014) 1535-1551.

[24] J. Quinta da Fonseca, E.C. Oliver, P.S. Bate, P.J. Withers, Mater. Sci. Eng. A. 437 (2006) 26-32.

[25] H. Wang, J. Zhou, J. Appl. Crystallogr. 38 (2005) 830-832.

[26] T. Ungár, A. Borbély, Appl. Phys. Lett. 69 (1996) 3173.

[27] P. Scardi, M. Leoni, R. Delhez, J. Appl. Crystallogr. 37 (2004) 381-390.

[28] G. Ribarik, N. Audebrand, H. Palancher, T. Ungar, D. Louer, J. Appl. Crystallogr. 38 (2005) 912-926.

[29] Y.H. Dong, P. Scardi, J. Appl. Crystallogr. 33 (2000) 184-189.

[30] H. Chen, Y.L. Yao, J.W. Kysar, I.C. Noyan, Y. Wang, Int. J. Solids Struct. 42 (2005) 3471-3485.

[31] H. Savaloni, M. Gholipour-Shahraki, M. a Player, J. Phys. D. Appl. Phys. 39 (2006) 2231-2247.

[32] U.F. Kocks, H. Mecking, Prog. Mater. Sci. 48 (2003) 171-273.

[33] H. Conrad, Prog. Mater. Sci. 26 (1981) 123-403.

[34] H. Mecking, U.F. Kocks, Acta Metall. 29 (1981) 1865.

[35] W.-S. Lee, C.-F. Lin, Mater. Sci. Eng. A. 308 (2001) 124-135.

[36] S. V. Raj, G.M. Pharr, Mater. Sci. Eng. 81 (1986) 217-237.

[37] T.H. Simm, DIPPA: v1_beta, http://dx.doi.org/10.5281/zenodo.59156, 2016.

[38] T.H. Simm, The Use of Diffraction Peak Profile Analysis in Studying the Plastic Deformation of Metals, PhD thesis, University of Manchester, 2012.

[39] G.K. Wertheim, M.A. Butler, K.W. West, D.N.E. Buchanan, Rev. Sci. Instrum. 45 (1974) 1369.

[40] H.P. Klug, L.E. Alexander, X-ray diffraction procedures for polycrystalline and amorphous materials., Wiley, 1974.

[41] A.R. Stokes, A.J.C. Wilson, Proc. Phys. Soc. 56 (1944) 174-181.

[42] B.I. Rodgers, P.B. Prangnell, Acta Mater. 108 (2016) 55-67.

[43] Y.T. Prabhu, K.V. Rao, World J. Nano Sci. Eng. 4 (2014) 21-28.

[44] N. Naga Krishna, R. Tejas, K. Sivaprasad, K. Venkateswarlu, Mater. Des. 52 (2013) 785-790.

[45] K.P. Shejale, D. Laishram, M.S. Roy, M. Kumar, R.K. Sharma, Mater. Des. 92 (2016) 535-540.

[46] G. Zhang, Z. Zhou, K. Mo, Y. Miao, S. Li, X. Liu, M. Wang, J.-S. Park, J. Almer, J.F. Stubbins, Mater. Des. 98 (2016) 61-67.

[47] M. Imam, V. Racherla, K. Biswas, Mater. Des. 64 (2014) 675-686.

[48] E. Wu, E.M.A. Gray, E.H. Kisi, J. Appl. Crystallogr. 31 (1998) 356-362.

[49] R. Delhez, T.H. de Keijser, E.J. Mittemeijer, Fresenius Z Anal Chem. 312 (1982) 1-16.

[50] I. Groma, Phys. Rev. B. 57 (1998) 7535-7542.

[51] U.F. Kocks, Mater. Sci. Eng. A. 317 (2001) 181-187. 
[52] T. Ungár, J. Gubicza, P. Hanak, I. Alexandrov, Mater. Sci. Eng. A. 319-321 (2001) 274-278.

[53] F. HajyAkbary, J. Sietsma, A.J. Böttger, M.J. Santofimia, Mater. Sci. Eng. A. 639 (2015) 208-218.

[54] M. Wilkens, J. Appl. Crystallogr. 12 (1979) 119-125.

[55] M. Zehetbauer, T. Ungár, R. Kral, A. Borbély, E. Schafler, B. Ortner, H. Amenitsch, S. Bernstorff, Acta Mater. 47 (1999) 1053-1061.

[56] X. Feaugas, Acta Mater. 47 (1999) 3617-3632.

[57] T.S. Byun, E.H. Lee, J.D. Hunn, J. Nucl. Mater. 321 (2003) 29-39.

[58] T. Ungár, Icotom 14 Textures Mater. Pts 1And 2. 503-504 (2006) 133-140.

[59] S. Mehta, S.K. Varma, J. Mater. Sci. 27 (1992) 3570-3574.

[60] V. Fitseva, S. Hanke, J.F. dos Santos, P. Stemmer, B. Gleising, Mater. Des. 110 (2016) 112-123.

[61] D. He, J. Zhu, S. Zaefferer, D. Raabe, Mater. Des. 56 (2014) 937-942.

[62] O. Morozov, V. Zhurba, I. Neklyudov, O. Mats, A. Rud, N. Chernyak, V. Progolaieva, Nanoscale Res. Lett. 10 (2015) 154.

[63] G. Ribárik, J. Appl. Crystallogr. 34 (2001) 669-676.

[64] P. Scardi, M. Leoni, Acta Crystallogr. Sect. A Found. Crystallogr. 58 (2002) 190-200.

[65] A. Borbely, J. Dragomir-Cernatescu, G. Ribarik, T. Ungar, J. Appl. Crystallogr. 36 (2003) 160-162.

[66] T. Ungár, G. Tichy, Phys. Status Solidi. 171 (1999) 425-434.

[67] I.C. Dragomir, T. Ungar, J. Appl. Crystallogr. 35 (2002) 556-564.

[68] T.H. de Keijser, J.I. Langford, E.J. Mittemeijer, A.B.P. Vogels, J. Appl. Crystallogr. 15 (1982) 308-314.

[69] C.R. Houska, R. Kuzel, 6: Classical treatment of line profiles influenced by strain, small size, and stacking faults, in: R.L. Snyder, F. J., H.J. Bunge (Eds.), IUCR Monogr. Crystallogr. Defects Microstruct. Analyis by Diffr., Oxford University Press, Oxford, 1999: pp. 264-317.

[70] M. Wilkens, Fundamental Aspects of Dislocation Theory, Vol. II, Natl. Bur. Stand. (US)Spec. Publ. No. 317, Washington DC USA, 1970.

\section{A. Appendix: Fourier Methods}

In this section the analytical procedure used for the Fourier methods are described in more detail.

The intensity values in a diffraction pattern are dependent on a smoothly varying function called the LorentzPolarisation factor [3]. This can be corrected for but because it only has a significant effect when the peaks are very wide, this was neglected. The Fourier coefficients of a diffraction peak must be determined between a constant separation $(\Delta K)$. The value used was $\Delta K=0.04 \times 10^{-10} \mathrm{~m}$, which gave enough information on the peak shape without being too big to be affected significantly by the overlapping of other peaks. For removing instrumental broadening the Stokes correction [41] was used. The method was found to be very useful in removing instrumental broadening; however, it can on some occasions become unstable when the physical broadening is low. The level of the background has an important influence on the Fourier coefficients and 
consequently the results. This is particularly the case at low Fourier lengths, which can result in the forbidden 'hook-effect' or a big drop in coefficients. However, there is no way of determining beforehand what the background level should be. The method adopted was to fit a straight line to the first few Fourier coefficients of the measured, instrumental and physically broadened peaks. The background level used is when these straight lines best fit the data. If this gives results that are inconsistent with other results and expectations (e.g. the 'hook-effect' for size FCs or unrealistic values), the procedure is repeated by adjusting the background manually and repeating.

The original method used by Warren and Averbach [3] to separate the size and strain coefficients was done by fitting the Fourier coefficients with different $n$ (or $L$ ) separately (or individually, or the INDI approaches in the terminology of this paper). However, it has become popular $[63,64]$ to fit to all the Fourier coefficients together, or even to fit to the intensity values of all peaks (the ALL approach in the terminology of this paper). For the different approaches slightly different equations and methodology are used. We are considering 3 methods to separate size and strain. In the standard Warren-Averbach formula, which we call the log-WA method (Equation A.1). To determine size and strain coefficients we can plot the log of the FCs against $g^{2}$ for different values of the Fourier length, the intercept and gradient give the size and strain coefficients respectively.

$$
\ln \left(A_{L}\right)=+\ln \left(A_{L}^{S}\right)-2 \pi^{2} g^{2} L^{2}\left\langle\varepsilon_{L}^{2}\right\rangle
$$

The linear form of the Warren-Averbach method, lin-WA (Equation A.2). The approach to finding size and strains is similar to the log form but the Fourier coefficients are plotted instead of the log of these.

$$
A_{L}=A_{L}^{S}\left(1-2 \pi^{2} g^{2} L^{2}\left\langle\varepsilon_{L}^{2}\right\rangle\right)
$$

The alternative method is given in Equation A.3. In this method the values of $L$ need to be converted to $L_{i}$ and then similar to other methods the size and strain can be found from the gradient and intercept of the log of the adjusted Fourier coefficients (on the $L_{i}$ scale rather than the $L$ scale).

$$
\begin{aligned}
& A^{D}\left(L_{i}, g_{i}\right)=A^{D}\left(L_{1}, g_{1}\right) \quad \text { with } L_{i}=\left(g_{1} / g_{i}\right) L_{1} \\
& \ln \left[A\left(L_{i}, g_{i}\right)\right]=\ln \left[A^{D}\left(L_{1}, g_{1}\right)\right]+\left(g_{1} / g_{i}\right) \ln \left[A_{s}\left(L_{1}\right)\right]
\end{aligned}
$$

The differences between the log-WA and alternative methods is highlighted in Figure 14. For the alternative method the Fourier length of higher order peaks is increased shifting them closer to the coefficients of the lower order. In this case they overlap meaning there is no size broadening. 

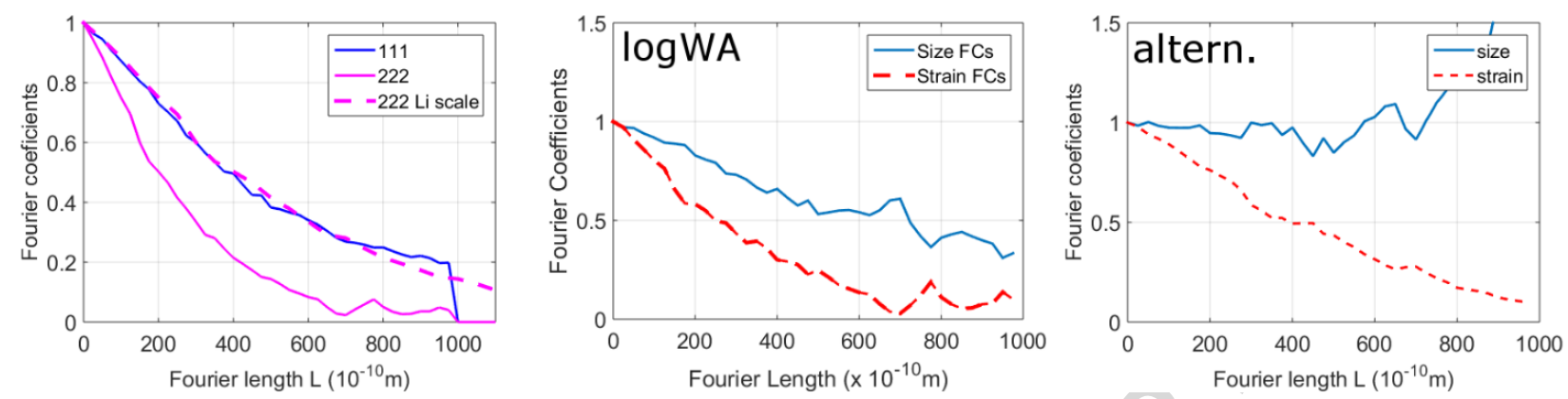

Figure 14, the Fourier coefficients of SS-316 at 10\% applied strain (left) including the adjusted Fourier coefficients of 222 for the alternative method. And right, the size and strain coefficients found using the log-WA method (equation A.1) and the alternative method (equation A.3).

\section{The Contrast Factor}

The type of dislocations present within a metal has an important influence on the broadening of a diffraction peak. This can be called the dislocation population, and provides details of the Burgers vector and slip plane, as well as the relationship between the dislocations line vector and Burgers vector $[23,65]$. The most common approach, is to assume the sample has a random texture (no preferred orientation) and that a particular slip system (with a particular Burgers vector and slip plane, e.g. \{111\} [110] for FCC metals) are equally populated. This approximation leads to a description for the contrast factor for a cubic material [66] as:

$$
\bar{C}=C_{h 00}\left(1-q H^{2}\right) \quad H^{2}=\frac{h^{2} k^{2}+h^{2} l^{2}+k^{2} l^{2}}{\left(h^{2}+k^{2}+l^{2}\right)^{2}}
$$

And for HCP alloys given by [67]:

$$
\bar{C}_{h k . l}=\bar{C}_{h k .0}\left(1+q_{1} x+q_{2} x^{2}\right) \quad x=\frac{2 l^{2}}{3(g a)^{2}}
$$

Where, $g=1 / d ; h, k$ and $/$ are the indices of the diffraction peak; $a$ one of the lattice parameters of the HCP unit cell. The value of $q$ (or $q_{1}$ and $q_{2}$ for HCPs) is used as fitting parameters to explain broadening heterogeneity (why broadening doesn't change smoothly with $g$ ). But it also has a physical meaning as it indicates the dislocation population, and in turn the dislocation population has an influence on the magnitude of $\bar{C}$. And so how the slip systems are determined can have a large effect on the value of the contrast factor. The method used to determine $C$ is similar to that used by Ungar and colleagues and is described in more detail in [38]. Other than the 'individual' methods and the alternative method, all methods are used with the contrast factor as a fitting parameter. The q-values for the FCC analysis were obtained from a preliminary 'ALL' fit of the Fourier coefficients, for both 'ALL' and 'INDI' methods.

\section{The INDI method of fitting}

\section{Size Coefficients}

When using the 'INDI' methods and alternative method, the size coefficients were fitted to the following equation: 
This is a simplification that has been used by others [68], but it can lead to errors in size values, and even strain values depending on the fitting method [69]. The reason for its use is that the size coefficients of FCC samples were not determined with enough accuracy to use a log-normal distribution and adequately determine the difference between the variance and mean; In some cases, the size coefficients became extremely noisy at values lower than 0.5 .

In some of the specimens the forbidden 'hook-effect' [3] of the size coefficients was observed irrespective of the background level. This was corrected for by fitting a curve to the coefficients in the non-hooked region, extrapolating this to the $y$-axis and then normalising. The extent of the 'hook-effect' was larger for the titanium samples than the FCC samples and more at low applied strains. This effect may be due to instrumental effects, but could also be due to the arrangement of dislocations into small angle grain boundaries [54].

\section{Strain Coefficients}

The strain coefficients were fitted using the description of Groma [50], but simplified to ignore terms with exponents of $L$ of 4 or higher. The equation was fitted over a distinct range of the Fourier length $(L)$ using the Krivoglaz-Wilkens plot. We use $L$ between approximately $100 \AA$ to $300 \AA$ Á, although this was adjusted slightly if there was a clear linear relationship slightly outside this range.

\section{The ALL Methods of fitting}

In the ALL method all the Fourier coefficients of all peaks, are fitted together. To do this the Fourier coefficients are fitted to a function that describes both size and strain distributions and how they are combined. This functions must be valid over the entire range of $L$ used. Because of this we use different equations to describe the strain and size broadening than in the INDI method. The Wilkens function must be used instead of Groma's description, because it doesn't increase at high values of $L$ and therefore better describes the strain coefficients over the whole range of $L$.

$$
\ln A_{D}(L)=-\rho B L^{2}\left(\frac{1}{d^{2}} \bar{C}\right) f\left(\frac{L}{R_{e}^{*}}\right), R_{e}=0.5 \exp (-0.25) \exp (2) R_{e}^{*}
$$

where, $A_{D}$ are the distortion Fourier coefficients, $\rho$ the dislocation density, $C$ the contrast factor, $L$ the Fourier length, and $R_{e}$ the outer cut-off radius. The term on the right is called the Wilkens function [70] and is given approximately by [64]:

$$
f^{*}(x)=\left\{\begin{array}{lll}
-\ln x+7 / 4-\ln 2+\frac{x^{2}}{6}-\frac{32 x^{3}}{225 \pi} & \text { for } & x \leq 1 \\
\frac{256}{45 \pi x}-\left(\frac{11}{24}+\frac{\ln 2 x}{4}\right) x^{-2} & \text { for } & x>1
\end{array}\right.
$$

A number of equations can be used to describe size broadening [64], of these we use a log-normal description because it has been shown to be a good approximation to the size distribution of many metals [11]. The use of 
this equation avoids the simplifications of the equation used in the 'INDI' method, which in the case of the ALL method would also influence the strain results. The size coefficients were described using a lognormal distribution given by [64].

When using the ALL method, in some cases the separation of the dislocation density and the dipole character was problematic. In particular, the values of the dipole character could tend towards very low values and the dislocation density to high values (this was especially the case for nickel). To try to reduce this error we could introduce tighter fitting boundaries. But the problem with this is can end up forcing the result we want. 

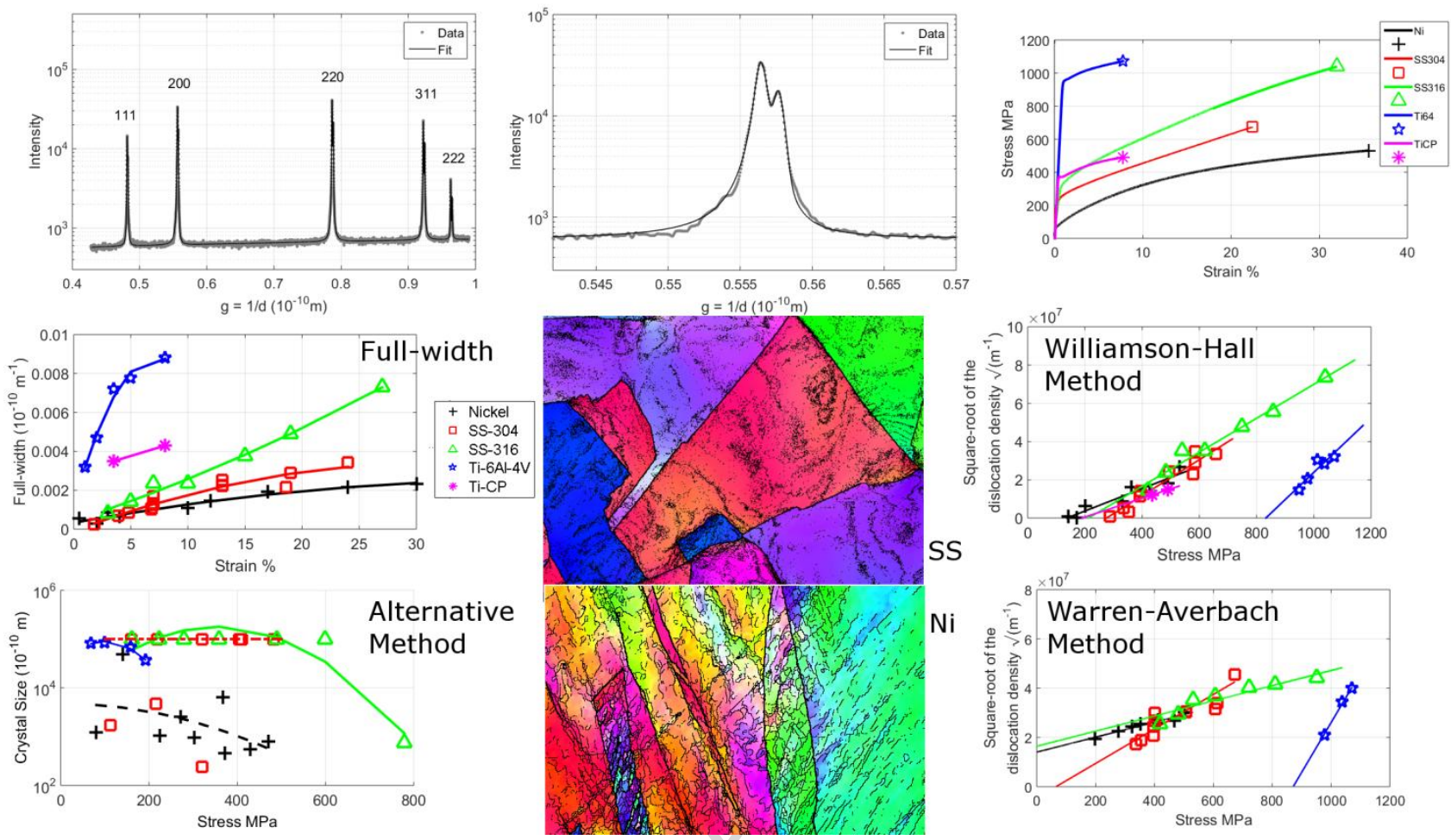

Graphical abstract 
Highlights

- The Williamson-Hall method produced dislocation density values more consistent with expectations than the Warren-Averbach method.

- The procedure for separation of different components; instrumental from real, size from strain, and dislocation density from arrangement; can cause errors most notably for Fourier methods.

- The size value obtained by most diffraction peak profile analysis methods is not helpful in quantifying a deformed metal.

- The alternative method, an exception to the above, provides size values that can be related to an effective crystal size in deformed metals. 